\title{
Quociente de inteligência de crianças e adolescentes obesos através da escala Wechsler*
}

\author{
Intelligent quotient of obese children and adolescents \\ by the Weschler scale
}

\author{
Alba L. R. Campos, Dirce M. Sigulem, Denise E. B. Moraes, Arlete M. S. Escrivão e Mauro \\ Fisberg
}

Departamento de Pediatria da Universidade Federal de São Paulo. São Paulo, SP - Brasil

\begin{abstract}
Resumo
Foram estudadas as características intelectuais de 65 crianças obesas, de 8 a 13 anos e 11 meses, comparativamente às de 35 crianças eutróficas, através da Escala de Inteligência Wechsler para Crianças. Os dois grupos foram pareados de acordo com a faixa etária, série escolar e nível socioeconômico. O grupo de obesos foi composto por pacientes atendidos pelo Departamento de Pediatria da Universidade Federal São Paulo, e o grupo-controle, por crianças pertencentes a escolas públicas. O grupo de eutróficos apresentou significantemente melhor desempenho no teste de inteligência do que o grupo de obesos (Quociente de inteligência -QI médio $91 \times 85 ; \mathrm{p}<0,05)$. Crianças eutróficas revelaram maior amplitude de interesses e capacidade de adaptação social e, melhor velocidade e destreza. Houve correlação positiva entre o nível de renda, a relação peso/estatura e o QI. Apesar de todos os resultados médios de QI apresentarem dados consistentemente favoráveis aos eutróficos, não se pode afirmar total superioridade desse grupo em virtude da ampla gama de fatores emocionais intervenientes no processo de inteligência, não controlados pelo estudo.
\end{abstract}

Escala de Wechsler. Obesidade.

\begin{abstract}
The intellectual characteristics of 65 obese children and adolescents (weight for height $\geq 140 \%$ ), aged 8 to 13 years and 11 months, were compared to those of 35 eutrophic children and adolescents (weight for height between 90 and $110 \%$; and stature for age $>95 \%$ ) of the same age group, utilizing the Wechsler Intelligence Scale for Children - WISC. Children and adolescents of the two groups were paired according to age groups, schooling level and socioeconomic condition. The obese group was composed of new patients assisted at the Department of Pediatrics of the Federal University of S. Paulo (Escola Paulista de Medicina), Brazil. The control group was made up of children from public primary schools, from the same geographical area as those studied. The eutrophic group presented significantly better performance in the intelligence test (Intelligence Quotient - IQ) than the obese group (average IQ - 91 x 85; p < 0.05). Eutrophic children and adolescents revealed
\end{abstract}

\footnotetext{
* Pesquisa subvencionada pela CAPES. Parte de Dissertação de Mestrado apresentada por Alba L. R. Campos à Universidade Federal São Paulo, 1994

Correspondência para / correspondence to: Alba L. R. Campos - Rua Cayowáa, 674 - 05018-000 - São Paulo, SP, Brasil. Fax: (011) 607.6710

Edição subvencionada pela FAPESP. Processo 95/2290-6.

Recebido em 8/6/1995. Aprovado em 26/10/1995.
} 
a wider range of interests, better capacity for social adaptability as well as greater speed and dexterity. Although weak, there was a positive correlation between income level, weight/stature relation (W/S) and IQ. There was no correlation between IQ and level of schooling. The eutrophic boys from higher income levels showed better perceptual and spacial organizing ability and a wider range of interests than those from the lower income groups. In spite of the fact that all the average IQ results presented consistently favored the eutrophic in relation to the obese, it is not possible to confirm one group's superiority over the other, due to the wide range of intervenient factors involved in the intelligence process.

\section{Wechsler scales. Obesity.}

\section{INTRODUÇÃO}

A obesidade é reconhecida desde a Segunda Guerra Mundial como um problema de saúde pública, representando atualmente o principal distúrbio nutricional dos países industrializados. $\mathrm{O}$ aumento dos casos de excesso de peso é descrito também em países em desenvolvimento, onde coexiste com a desnutrição ${ }^{12}$. A obesidade pode ser considerada como uma síndrome, definida pelo aumento de tecido adiposo em relação à massa corporal magra e determinada, na maior parte dos casos, por inadequação entre ingestão alimentar e dissipação de calor ou energia ${ }^{5}$. Na criança em idade escolar, a maioria dos estudos define obesidade sempre que a relação peso/estatura (P/E) excede $20 \%$ do padrão desejável para a idade e o sexo ${ }^{8}$. Trabalhos realizados na faixa etária pediátrica mostram que esta relação tem pouca associação com o índice de massa corporal, comumente utilizado para a classificação nutricional da obesidade em adultos ${ }^{5}$.

Fisberg $^{7}$ (1993) afirma que a obesidade exógena constitui $95 \%$ dos casos registrados e atendidos em ambulatórios específicos de atendimento a distúrbios nutricionais com excesso de peso, sendo esse o foco de atenção do presente trabalho.

Os fatores relacionados à obesidade exógena são inúmeros e se sobrepõem fazendo com que esta situação nutricional seja considerada como de origem multifatorial.

As questões emocionais que cercam a obesidade são comumente citadas na literatura internacional; todavia os aspectos intelectuais da criança e do adolescente obesos não foram ainda estudados. Alguns trabalhos mostram investigações realizadas em grandes amostras, relacionando obesidade com performance intelectual reduzida, em adultos jovens 9,10,20,21. Entre as características psicológicas de crianças e adolescentes obesos são encontradas freqüentemente a imaturidade, a infantilização, a dependência, a passividade, a baixa auto-estima, a falta de iniciativa e a dificuldade de adiar gratificações ${ }^{2,4,6,13,14}$. Estas características podem determinar alterações importantes na dinâmica psicossocial da criança obesa, e modificar sua performance intelectual. Baseados nesses fatores, decidiu-se investigar se esses aspectos se associam a menores escores intelectuais analisados por meio de teste de inteligência. Os resultados foram comparados com os de grupos de crianças e adolescentes eutróficos, pareados por idade, escolaridade e nível social e econômico, afim de se verificar as diferenças entre essas duas populações.

\section{MATERIAL E MÉTODO}

Foi feito estudo transversal, com população de escolares obesos e eutróficos, no período compreendido entre 1991 e 1993. Caracterizaram-se como obesos os pacientes que apresentaram relação peso para estatura igual ou superior a $140 \%(\mathrm{P} / \mathrm{E} \approx 140 \%)$, do padrão do National Center for Health Statistics (NCHS) (OMS ${ }^{16}$, 1983). Este grupo foi constituído por 65 crianças e adolescentes ( 36 do sexo masculino e 29 do sexo feminino), na faixa etária de 8 a 13 anos e 11 meses. O grupo foi formado com pacientes novos atendidos pela Disciplina de Nutrição e Metabolismo do Departamento de Pediatria da Universidade Federal de São Paulo. O grupo-controle foi composto por 35 crianças eutróficas (18 do sexo masculino) de escolas públicas de primeiro grau, de acordo com as seguintes características antropométricas: peso para a estatura entre 90 a $110 \%$ $(\mathrm{P} / \mathrm{E}>90$ e $<110 \%)$ e estatura para a idade maior ou igual a $95 \%$ ( $\mathrm{E} / \mathrm{I} \approx 95 \%$ ), utilizando-se os padrões de referência do NCHS (OMS $\left.{ }^{16}, 1983\right)$. As duas amostras foram pareadas individualmente nas variáveis idade, escolaridade e faixa de renda. As crianças dos dois grupos foram submetidas, individualmente, à aplicação da "Wechsler Intelligence Scale for Children" (WISC) ${ }^{22}$. 
Neste teste, todos os itens de determinado tipo são agrupados em subtestes e dispostos em ordem crescente de dificuldade. O resultado bruto de um sujeito, em cada subteste é convertido em resultados-padrão, normalizados de acordo com o grupo de idade. Existem tabelas com esses resultados-padrão para cada intervalo de 4 meses, entre as idades de 5 a 15 anos, os quais são expressos em função da distribuição com média 10 e desvio-padrão de 3 pontos. Os resultados-padrão de subtestes são somados e convertidos num Quociente de Inteligência (QI) de desvio com média $100^{1}$. O WISC foi aplicado, obedecendo as instruções específicas contidas em seu manual que também orientou a correção e análise dos resultados ${ }^{22}$. O teste utilizado é adaptado para a população nacional e é de uso corrente nos ambulatórios de nutrição e psicologia da Universidade Federal de São Paulo (UNIFESP) ${ }^{22}$. Para análise dos resultados foram utilizados testes estatísticos, não paramétricos, de acordo com as variáveis em estudo, a natureza da distribuição dos valores e a variabilidade das medidas estudadas. Foram aplicados os seguintes testes: Mann-Whitney para duas amostras independentes, análise da variância por postos de Kruskal-Wallis, Quiquadrado para tabelas $2 \times 2$, coeficiente de correlação de Spearman ${ }^{19}$. Para todos os testes fixou-se em $5 \%(\mathrm{p}<$ 0,05) o nível para rejeição da nulidade.

\section{RESULTADOS E DISCUSSÃO}

Encontramos diferenças estatisticamente significantes nos resultados de QI (Figura 1), sendo que o grupo eutrófico apresentou melhor desempenho no teste de inteligência do que o grupo de obesos. O QI médio dos eutróficos $(91,24)$ se localizou dentro da faixa de Normalidade (90-109), enquanto que o dos obesos ficou dentro da classificação "médio inferior" (85,97). Analisando a amplitude de QI constatou-se que o menor QI do grupo de obesos foi 56 , contra 74 do grupo de eutróficos, ou seja, diferença de aproximadamente 20 pontos o que situou o menor resultado dos obesos na classificação "débil mental", enquanto que o dos eutróficos se localizou na faixa limítrofe, de acordo com a classificação do manual do teste ${ }^{22}$.

Também se observou diferença relevante ao se comparar o maior QI dos dois grupos: 118 para os eutróficos e 107 para os obesos.

No caso dos adultos, a literatura levanta como possível explicação, para melhor desempenho de eutróficos, nos testes de inteligência, a hipótese de que existem fatores causais comuns à obesidade $\mathrm{e}$ ao desempenho intelectual reduzido ${ }^{10,20}$. Tais fatores são de natureza genética ou ambiental. No caso das crianças e adolescentes, de acordo com nossa experiência fundamentada na problemática psicológica da criança obesa ${ }^{4,6,14}$, optou-se por buscar explicações que caminham pela esfera emocional, já que a função intelectual não pode ser desvinculada de outras funções psíquicas e compreendida fora do sistema dinâmico e complexo do qual faz parte.

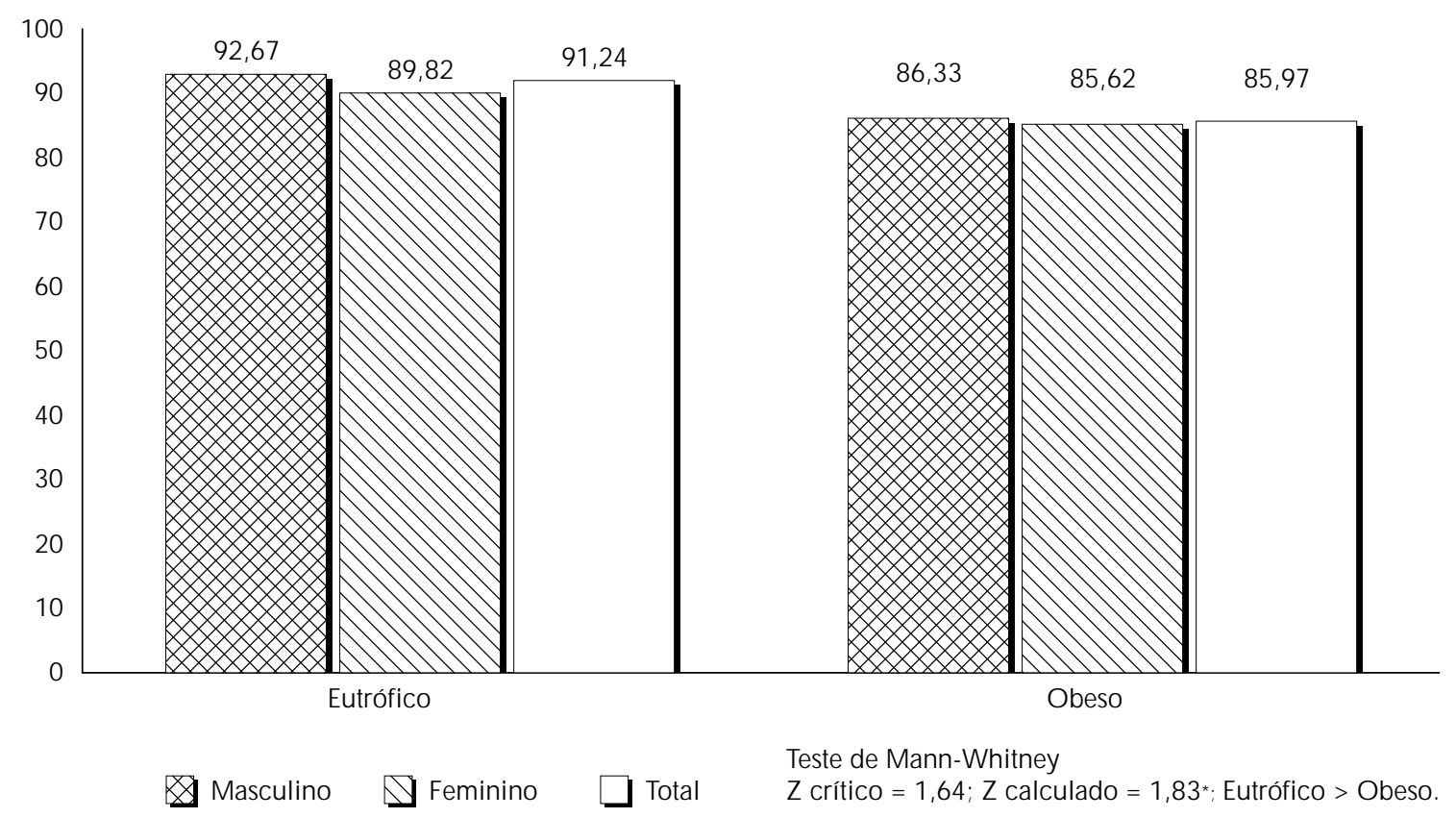

Figura 1 - Crianças e adolescentes, eutróficos e obesos do sexo masculino e do feminino, segundo os valores do Q uociente de Inteligência Total (Q I Total) 
A ênfase sobre os motivos psicológicos e emocionais que podem levar a criança a ser obesa recaem sobre as falhas estruturais da relação mãe/ filho, vínculo através do qual a personalidade se desenvolve ${ }^{14,17}$. Também os processos cognitivos e intelectuais dependem das experiências emocionais precoces. Várias teorias (especialmente a psicanalítica) confirmam essas suposições. Luzuriaga $^{11}$ considera que a inteligência pode ser
$\operatorname{Birch}^{3}$ faz menção à interação e estimulação propiciadas pelas mães de crianças obesas, fazendo referência ao reduzido número de respostas diretas ou indiretas durante a realização de jogos, apesar de haver maior solicitação por parte das crianças, em comparação às fornecidas pelas mães de crianças magras, mostrando que as primeiras, oferecendo menor estimulação prejudicam o desenvolvimento intelectual de seus filhos.

Tabela 1 - Crianças e adolescentes do sexo masculino e do feminino, segundo o grupo e os valores abaixo ou iguais e acima do Quociente de Inteligência (Q I) médio.

\begin{tabular}{|c|c|c|c|c|}
\hline \multirow[t]{2}{*}{ Grupo } & \multicolumn{4}{|c|}{ QI } \\
\hline & < M édia & < Média & Total & $\%<$ M édia \\
\hline Eutrófico & 16 & 19 & 35 & 45,7 \\
\hline O beso & 41 & 24 & 65 & 63,1 \\
\hline Total & 57 & 43 & 100 & 57,7 \\
\hline
\end{tabular}

Tabela 2 - Médias de resultados de acordo com o subteste, o Q I total e o estado nutricional, por sexo.

\begin{tabular}{|c|c|c|c|c|}
\hline & \multicolumn{2}{|c|}{ Eutrófico } & \multicolumn{2}{|c|}{ Obeso } \\
\hline & Fem & Masc & Fem & Masc \\
\hline Informação & $8,47^{a}$ & $8,94^{a}$ & 7,03 & 7,58 \\
\hline Compreensão & 5,29 & $6,94^{b}$ & 5,79 & $6,17^{b}$ \\
\hline Aritmética & 8,24 & 10,06 & 7,90 & 8,67 \\
\hline Semelhanças & 9,41 & 9,44 & 8,93 & 8,92 \\
\hline Números & 9,94 & 9,22 & 9,03 & 8,47 \\
\hline Complet. Figuras & 7,12 & 8,44 & 6,86 & 7,31 \\
\hline Arranjo figuras & 8,88 & 8,17 & 8,17 & 8,08 \\
\hline Cubos & 8,88 & 10,18 & 8,83 & 8,92 \\
\hline Armar objetos & 7,71 & 8,89 & 7,24 & 7,67 \\
\hline Código & $12,06^{a}$ & $9,56^{a}$ & $10,07^{b}$ & 9,19 \\
\hline Q I total & $89,82^{a}$ & $92,67^{a}$ & 85,62 & 86,33 \\
\hline QI tot. (masc +fem) & \multicolumn{2}{|c|}{91,24} & \multicolumn{2}{|c|}{85,97} \\
\hline
\end{tabular}

$\mathrm{a}=\mathrm{p}<0,05$ (masculino $x$ feminino)

$b=p<0,05$ (eutrófico $x$ obeso)

usada contra si mesma. É como se a inteligência, com a finalidade de não conhecer conteúdos dolorosos, fosse empregada na própria destruição. Dessa forma as funções intelectuais, tais como percepção dos objetos externos e internos, memória, concentração e outras, sofrem a ação dos mecanismos defensivos (negação, racionalização e outros) a tal ponto que as possibilidades de desenvolvimento intelectual e afetivo do indivíduo são destruídas. As experiências emocionais precoces, nas quais a relação com a mãe tem fundamental importância, influenciam a formação do pensamento, nos processos de simbolização e discriminação da criança ${ }^{15}$.
$\mathrm{Na}$ obesidade, são relatadas freqüentemente características como dependência, agressividade mal elaborada e dificuldade de se sentir em igualdade de condições para competir com indivíduos de seu meio ${ }^{4,6,14,18}$. É provável que essas características associadas ao funcionamento psiquíco mais lento e mais primitivo do indivíduo obeso, não contribuem para que ele tenha como foco de interesse a ambição intelectual que o levaria a investir mais nesse aspecto.

Quando se separa os dois grupos estudados em valores menores ou maiores/iguais à faixa média de QI (Tabela 1), observa-se que percentualmente 

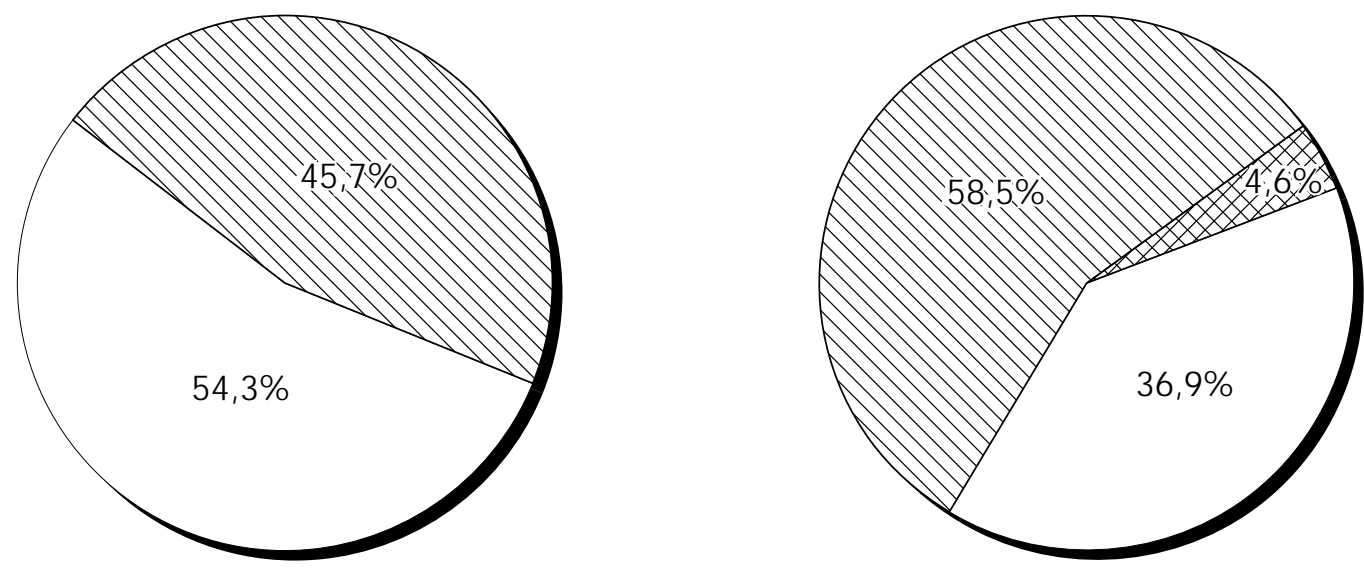

$<70$

$70-89$

】 $>=90$

Figura 2 - Distribuição dos Q Is das crianças e adolescentes, do sexo masculino e feminino, segundo o grupo e de acordo com a classificação da inteligência

existe diferença, já que $63,1 \%$ dentre os casos do grupo de obesos apresentam resultados de QI total inferior à faixa média ${ }^{22}$, contra $45 \%$ do grupo eutrófico. Entretanto, o teste do quiquadrado não apresentou diferença significante. Apesar disso, vale ressaltar algumas diferenças. Assim, no grupo de obesos (Figura 2), 4,6\% dos casos apresentaram resultados de QI total inferiores a 70, o que corresponde à debilidade mental. Entre os eutróficos não se encontrou nenhum caso de debilidade mental, mas por outro lado, o resultado médio encontrado foi superior em $2,8 \%$ dos casos, ao passo que no grupo de obesos nenhum caso atingiu tais escores. Neste grupo, $24,6 \%$ das crianças conseguiram QIs classificados na faixa limítrofe (QI 70-79), contra 14,3\% dos eutróficos. A classificação médio-inferior (80-89) foi obtida por 33,8\% dos obesos e $31,4 \%$ dos eutróficos. Nesta faixa os resultados foram muito similares nos dois grupos. $\mathrm{Na}$ faixa média (90-109) se localizaram 36,9\% dos obesos contra $51,5 \%$ dos eutróficos, o que significa que mais da metade destes últimos, estudados no presente trabalho apresentaram QI médio, o que só ocorre com aproximadamente um terço dos obesos. Esses resultados explicam, em parte, a diferença a favor dos eutróficos no resultado geral.

O grupo de obesos revelou menor amplitude de conhecimentos e estreitamento do campo de interesses em relação ao grupo eutrófico (Tabela 2).
Oliveira e Santiago ${ }^{15}$, relacionam inúmeros fatores que contribuem para a falta ou diminuição da disponibilidade da criança em relação às informações do meio ambiente. Tensões na família, rivalidade excessiva, componentes de ciúme e inveja, acentuada exigência ou desinteresse dos pais, dificuldade de enfrentar a falta de gratificação imediata de seus impulsos, e outras, concorrem em maior grau para o desenvolvimento de conflitos intrapsíquicos na criança, dependendo de suas fantasias. São essas que, freqüentemente, desviam sua atenção das informações apresentadas pela realidade para outras situações externas ou internas que lhe são mais importantes. Estudos anteriores mostram que esses fatores estão presentes na criança obesa ${ }^{4,6,14}$. Talvez eles tenham influído negativamente, apesar de não permitirem estabelecer relações de causa e efeito, já que as questões emocionais não foram controladas no presente estudo.

Menores condições de velocidade e destreza também foram encontradas no grupo de obesos, quando comparados ao grupo eutrófico. A lentidão característica dos obesos pode ter se refletido nos resultados.

Quando foram correlacionados os valores de QI com a adequação de $\mathrm{P} / \mathrm{E}$, foi encontrada correlação inversa significante, embora pequena $(r=0,21)$. A literatura mostra a existência de relação inversa entre os índices de obesidade e os resultados dos testes 
de inteligência em populações de adultos jovens ${ }^{9,21}$.

Tanto o grupo de obesos quanto o de eutróficos manifestaram, em algum grau, certa problemática emocional e não revelaram diferença quanto ao potencial intelectual.

A importância do presente estudo, em relação à criança e ao adolescente obeso, é ampliar o conhecimento sobre seu funcionamento do ponto de vista intelectual e cognitivo, não como forma de estigmatizá-la, mas para que se compreenda o porquê desses resultados e assim se enfatize a necessidade de prevenção do distúrbio e se encaminhem as soluções passando também por uma abordagem relacionada com a questão da aprendizagem. Desta forma quando não for possível

\section{REFERÊNCIAS BIBLIOGRÁFICAS}

1. ANASTASI, A. As escalas Wechsler e a mensuração da inteligência adulta. In: Anastasi, A. Testes psicológicos. São Paulo. EPU, 1977. p. 323-51.

2. BANIS, H.P. et al. Psychological and social adjustement of obese children and their families. Child Care Health Dev., 14:157-73, 1988.

3. BIRCH, L.L. et al. Mother-child interaction pattern and the degree of fatness in children. J. Nutr. Educ., 13:17-21, 1981.

4. CAMPOS, A.L.R. Aspectos psicológicos da obesidade. Pediatr. Mod., 29:129-30, 1993.

5. FISBERG, M. et al. Obesidade na infância e adolescência. JBM, (6):30-5, 1992.

6. FISBERG, M. et al. Infantile obesity: a multidisciplinary approuch. Int. Child Health., 4(3): 77-82, 1993.

7. FISBERG, M. Obesidade na infância e adolescência. Pediatr. Mod., 29: 103-8, 1993.

8. KNEEBONE, M. Childhood obesity - the diagnosis and manengement. Aust. Fam. Physician., 19: 367-70, 1990.

9. KREZE, A. et al. Relationship between intelligence and the relative prevalence of obesity. Hum. Biol., 46:109-13, 1974.

10. LISSAU-LAND-SORENSEN, I. \& SORENSEN, T.I.A. The effect of parents' care on risk of becoming overweight in adulthood. Int. J. Obes., 16 (Suppl):244, 1992. (Abstract)

11. LUZURIAGA, I. La inteligencia contra si misma. Buenos Aires, Ed. Psique, 1987.

12. MARDONES, M.A. \& ATALAH, E. Prevalencia y factores condicionantes de la obesidad en adolecentes del sexo feminino. Rev. Chil. Pediatr., 58:311-6, 1987. prevenir, e o distúrbio já estiver instalado, deve-se ter a preocupação de aprender maneiras de desenvolver o potencial intelectual que eles revelam, para não contribuir para estabelecer o estigma.

\section{AGRADECIMENTOS}

Ao Professor Fernando José de Nóbrega, Christianne Nascimento, Marcel Chaves de Castilho e Souza, Cecília Michiko Itami Forti, Clenivalda França dos Santos, Ivone da Conceição Gomes e Marcos Rosa de Lima pela ajuda e apoio nas várias fases do trabalho. Aos Professores Neil Ferreira Novo e Yara Juliano pela colaboração e realização do tratamento estatístico.

13. NÓBREGA, F.J.\& CAMPOS, A.L.R. Fraco vínculo mãefilho: importante fator de risco da desnutrição. Ars Curandi, 26: 47-56, 1993.

14. NÓBREGA, F.J.\& CAMPOS, A.L.R. Distúrbios nutricionais e fraco vínculo mãe/filho. Rio de Janeiro, Revinter, 1996.

15. OLIVEIRA, L.G.M. \& SANTIAGO, M.D.E. Abordagem clínica da inteligência. In: Ancona-Lopez, M., org. Avaliação da inteligência I. São Paulo, EPU, 1987. p.96108.

16. ORGANIZACIÓN MUNDIAL DE LA SALUD. Medición del cambio del estado nutricional: directrices para evaluar el efecto nutricional de programas de alimentación suplementaria destinada a grupos vulnerables. Ginebra, 1983.

17. PIZZINATTO, V.T. Obesidade infantil. São Paulo, Ed Sarvier, 1992.

18. SAITO, M.I. et al. Visão multiprofissional do adolescente obeso. Pediatria (São Paulo), 7:210-4,1985.

19. SIEGEL, S. Estatística não-paramétrica. São Paulo, McGraw-Hill, 1975.

20. SONNE-HOLM, S. \& SORENSEN,T.I.A. Prospective study of attainment of social class of severy obese subjects in relation to parental social class, intelligence, and educacion, B.M.J., 292:586-9, 1986.

21. TEASDALE, T.W. et al. Intelligence and educational level in relation to body mass index of adult males. Hum. Biol., 64: 99-106, 1992.

22. WECHSLER, D. WISC. Escala de Inteligência Wechsler para Crianças. Manual de aplicação. Rio de Janeiro, CEPA, s.d. 